\title{
Glucagon-Like Peptide 1 Receptor Agonist (GLP1RA) Exposure and Outcomes in Type 2 Diabetes: A Systematic Review of Population-Based Observational Studies
}

\author{
Thomas M. Caparrotta (i) · Jack B. Templeton · Thomas A. Clay · \\ Sarah H. Wild · Rebecca M. Reynolds · David J. Webb • \\ Helen M. Colhoun
}

Received: January 21, 2021 / Accepted: January 30, 2021 / Published online: February 26, 2021

(C) The Author(s) 2021

\section{ABSTRACT}

Introduction: Glucagon-like peptide 1 receptor agonists (GLP1RAs) are licensed for the treatment of type 2 diabetes (T2D). They have been shown to be safe (from the cardiovascular (CV) perspective) and effective (in terms of glycaemia, and in some cases, reducing CV events) in extensive randomised controlled trials (RCTs). However, there remain concerns regarding the generalisability of these findings

Supplementary Information The online version contains supplementary material available at https:// doi.org/10.1007/s13300-021-01021-1.

T. M. Caparrotta $(\bowtie) \cdot$ J. B. Templeton ·

H. M. Colhoun

Institute of Genetics and Molecular Medicine,

University of Edinburgh, Edinburgh, UK

e-mail: tom.caparrotta@igmm.ed.ac.uk

T. A. Clay

NHS Lothian, Edinburgh Royal Infirmary,

Edinburgh, UK

S. H. Wild

Usher Institute, University of Edinburgh,

Edinburgh, UK

R. M. Reynolds · D. J. Webb

University of Edinburgh/British Heart Foundation Centre for Cardiovascular Science, Queen's Medical Research Institute, Edinburgh, UK

H. M. Colhoun

Department of Public Health, NHS Fife, Kirkcaldy, UK (to those ineligible for RCT participation) and about non-CV safety. For effectiveness, population-based pharmacoepidemiology studies can confirm and extend the findings of RCTs findings to broader populations and explore safety, for which RCTs are not usually powered, in more detail.

Method: We did a pre-planned and registered (PROSPERO registration CRD42020165720) systematic review of population-based studies investigating GLP1RA effectiveness and safety, following Meta-analyses Of Observational Studies in Epidemiology (MOOSE) guidelines.

Results: A total of 22 studies were identified (including 200,148 participants and 396,457 person-years of follow-up) exploring exposure to GLP1RA class, exenatide and liraglutide (the only individual drugs with treatment effect estimates identified) on mortality, cardiovascular disease (CVD), acute pancreatitis (AP), pancreatic cancer (PC), thyroid cancer (TC), acute renal failure (ARF), diabetic retinopathy (DR), breast cancer (BC) and hypoglycaemia. For $\mathrm{CV}$ and mortality outcomes, studies confirmed the associated safety of these drugs. For liraglutide, point estimate (PE) range (PER) major adverse cardiovascular events (MACE) (0.53-0.95) and PER heart failure (0.34-1.22) were similar in direction to the beneficial effect observed in RCTs for MACE but varied widely for heart failure. For safety outcomes, exposure was not associated with AP (PER 0.50-1.17), PC (PER 0.40-1.54), BC (PER 
0.90-1.51) or hypoglycaemia (PER 0.59-1.06). Only one study was identified exploring each of TC (no evidence of association, hazard ratio (HR) 1.46, 95\% confidence interval (CI) 0.98-2.19), renal outcomes (no evidence of association, HR $0.77,95 \%$ CI 0.42-1.41) and DR (no evidence of association, HR $0.67,95 \% \mathrm{CI}$ 0.51-0.90).

Conclusion: In T2D, GLP1RAs appear safe from the CV perspective and (for liraglutide) may have associated benefit in primary as well as secondary CVD prevention. For non-CV safety, GLP1RA exposure was not associated with an increased risk of AP, PC, BC or hypoglycaemia; the other outcomes had too few studies to draw firm conclusions and should be explored further.

Keywords: Drug safety; Effectiveness; GLP-1 receptor agonists; Type 2 diabetes

\section{Key Summary Points}

Glucagon-like peptide 1 receptor agonist (GLP1RA) are licensed for the treatment of type 2 diabetes (T2D).

For cardiovascular effectiveness, population-based pharmacoepidemiology studies can confirm and extend the findings of randomised controlled trials (RCTs) to broader populations not eligible for trial participation and explore safety, for which RCTs are not usually powered, in more detail.

We did a pre-planned and registered, agnostic systematic review asking: do the benefits of GLP1Ras in T2D extend to those ineligible for RCT participation, and are safety concerns which arose during the trials (or in post-marketing) detected, in population-based observational pharmacoepidemiology studies?

We considered and reported all clinical event-based outcomes for effectiveness and safety in studies which met our inclusion/exclusion criteria.
For cardiovascular (CV) and mortality outcomes, studies confirmed the associated safety of these drugs and, for liraglutide, correlated closely with the findings from RCTs, which may extend to primary CV disease prevention.

For safety outcomes, GLP1RA exposure was not associated with an increased risk acute pancreatitis, pancreatic cancer, breast cancer or hypoglycaemia. There were insufficient studies to draw conclusions with regards to thyroid cancer, renal outcomes and diabetic retinopathy.

\section{DIGITAL FEATURES}

This article is published with digital features, including a summary slide, to facilitate understanding of the article. To view digital features for this article go to https://doi.org/10.6084/ m9.figshare.13667132.

\section{INTRODUCTION}

Glucagon-like peptide 1 receptor agonists (GLP1RAs) are licensed for the treatment of type 2 diabetes (T2D). GLP1RAs have been shown to improve glycaemic control via regulation and restoration of incretin function, leading to enhancement of glucose-dependent insulin secretion, slowed gastric emptying, and reduction of postprandial glucagon secretion and food intake [1].

The clinical development programmes for these agents have robustly assessed the efficacy and preliminary non-cardiovascular $(\mathrm{CV})$ safety of GLP1RA drugs for use in people with T2D. Several have subsequently been assessed for CV safety in large outcome trials, which have yielded mixed results in terms of effect on cardiovascular disease (CVD; liraglutide, subcutaneously administered semaglutide and dulaglutide all reduce CVD, lixisenatide, extended release exenatide and orally administered semaglutide do not) although all appear to 
be safe from the CV perspective [2-8]. Nevertheless, it remains unclear whether the $\mathrm{CV}$ benefit (if any) of these medicines extends to people who were not eligible to participate in randomised controlled trials (RCTs).

Population-based database studies present more generalisable data which can help broaden and expand the findings of RCTs. Although many population-based studies apply appropriate study design and statistical methods for control of confounding, these studies are at higher risk of confounding and bias than RCTs because they are not randomised or blinded. For CV effectiveness and safety, they can confirm and extend the finding of RCTs to more heterogeneous populations, especially those ineligible for trial participation. RCTs are not powered to detect adverse events, thus it often remains unclear whether safety signals identified in trials are a true effect or due to chance. As such, for non-CV safety, large, observational studies remain an important tool to identify the risk of harm, and if detected, to identify characteristics of participants that are associated with risk.

Here we describe the conduct and findings of a systematic review of population-based, database effectiveness and safety studies of GLP1RAs in T2D, either as a class or as individual agents.

\section{METHODS}

We prospectively registered an impartial systematic review of population-based, observational studies examining effectiveness and safety of GLP1-RA agents in T2D (PROSPERO registration CRD42020165720, 16 January 2020). We hypothesised that these studies, if properly conducted, would show similar estimates for effectiveness and safety to those described in previous literature (Table 1).

We followed Meta-analyses Of Observational Studies in Epidemiology (MOOSE) guidelines for reporting the systematic review. In brief, we employed two search methodologies (maximal and targeted search) and interrogated the following research databases: Web of Science, Medline, EMBASE and EMCARE on Ovid, and CINAHL on EBSCO using the search criteria listed in the supplementary material (Table S1). We searched for studies published between 28 November 2006 (date of licensing of exenatide) and end of January 2020 . We eliminated duplicates using each study's unique identifier. Two reviewers (JT and TC; TC, medically qualified) independently applied the inclusion and exclusion criteria first to the title and then to the abstracts of potentially eligible studies (Table S2). We searched ENCePP, ClinicalTrials.gov and the EU clinical trials register as well as the references of eligible studies to identify further publications which may not have been identified in our searches. We only included English language studies because of resource limitations.

Each reviewer checked $10 \%$ of the other's title and abstract screening. If there was less than $95 \%$ agreement we pre-specified that the entire list would be re-screened. Third party arbitration of disputes was planned in the event of less than 95\% agreement. There was 100\% agreement on the $10 \%$ of included/excluded studies so this step was not necessary.

The studies eligible for inclusion were scored for quality (using the Downs and Black Checklist for Non-Randomised Studies) [9] but we did not employ a quality score cut-off for inclusion/ exclusion (Table S3). The quality score has been shown to have good inter-rater reliability [10].

We then extracted study information from the studies identified using a standardised data extraction template (Table S4). We included all reported clinical event outcomes but decided post hoc not to include the reporting of surrogate markers (continuous variables, e.g. glycated haemoglobin (HbA1c)).

All clinical event outcomes were included.

We tabulated the study information, point estimates and confidence intervals (CIs) (including relevant subgroup information) by outcome, so studies may appear in multiple tables (Tables S5, S7, S8, S9, S10, S11, S12, S13). For safety outcomes, where there were more than three studies exploring an outcome, we made forest plots for visual comparison.

We chose to describe studies with fewer than 5000 participants as small; 5000-20,000 participants as medium-sized; more than 20,000 as large. CI widths are described qualitatively. 
Table 1 Effectiveness and safety data from RCTs and other sources

\begin{tabular}{llll}
\hline Outcome & \multicolumn{2}{l}{ RCT (and other) evidence } & \\
\hline CVD & MACE (HR) & Non-fatal MI (HR) & Non-fatal stroke (HR) \\
& Exenatide QW [3] & & \\
& $0.91(0.83,1.00)$ & $0.95(0.84,1.09)$ & $0.86(0.70,1.07)$ \\
& Liraglutide [6] & & \\
& $0.87(0.78,0.97)$ & $0.88(0.75,1.03)$ & $0.89(0.72,1.11)$ \\
& CVD mortality (HR) & ACM (HR) & \\
Mortality & Exenatide QW [3] & & \\
& $0.88(0.73,1.05)$ & $0.86(0.77-0.97)$ & \\
& Liraglutide [6] & & \\
& $0.78(0.66,0.96)$ & $0.85(0.74,0.97)$ &
\end{tabular}

Pancreatic outcomes (PC and AP) There has been concern that GLP1RA exposure causes an increased risk of AP and PC. It is unclear whether this is an actual drug effect because of the small numbers arising in the clinical trial programmes and because T2D is a risk factor for AP and PC, in and of itself. Development of AP and PC is increased by diagnosis of DM, and the prevalence in the studies may be affected by reverse causality (where DM is caused by the presence of undiagnosed AP or PC). GLP1RAs have been found to induce chronic pancreatitis in rat models that can progress to cancer, via a suggested pathway involving ductal metaplasia and increased cell replication [47]

Thyroid cancer

Renal outcomes

Diabetic retinopathy

Breast cancer
Animal models have suggested that GLP1RA exposure may be associated with an increased risk of MTC [48]

There are no trials examining microvascular disease and GLP1RAs as a primary outcome

Recent trial data suggest that liraglutide, dulaglutide, semaglutide and lixisenatide may delay the onset of diabetic renal disease (through various secondary outcome measures) $[2,5-8]$

The mechanism by which GLP1RA exposure may influence DR is unclear. It may relate to rapid correction of hyperglycaemia, as has previously been reported with insulin [49] or may be related to the GLP1RA exposure itself

CVOT data suggest that semaglutide (subcutaneously administered) increased the incidence of DR (HR 1.76, 95\% CI 1.11-2.78) but not with orally administered semaglutide (although those with prevalent DR were excluded) $[4,7]$

For liraglutide there was a numerical, but not significant, imbalance towards more DR following exposure [6]

Dulaglutide appears to reduce DR [2]

A numerical imbalance of incident breast cancer was found in an RCT of liraglutide [50]. No mechanism has been elucidated for this putative adverse effect although there was no imbalance of breast cancer in LEADER [6] 
Table 1 continued

\begin{tabular}{ll}
\hline Outcome & RCT (and other) evidence \\
\hline Hypoglycaemia & $\begin{array}{l}\text { GLP1RA agents, either as a class or individually, do not appear to be associated with } \\
\text { an increased risk of severe hypoglycaemia. In the LEADER trial, liraglutide exposure } \\
\text { was association with less hypoglycaemia than placebo }(2.4 \% \text { vs. } 3.3 \%)[6]\end{array}$
\end{tabular}

$A C M$ all-cause mortality, $A P$ acute pancreatitis, $C V D$ cardiovascular disease, CVOT cardiovascular outcome trial, $D M$ diabetes mellitus, $D R$ diabetic retinopathy, GLPIRA glucagon-like peptide 1 receptor agonist, $H R$ hazard ratio, $L E A D E R$ Liraglutide Effect and Action in Diabetes: Evaluation of cardiovascular outcome Results, MACE major adverse cardiovascular events, $M I$ myocardial infarction, $M T C$ medullary thyroid cancer, $P C$ pancreatic cancer, $Q W$ once weekly, $R C T$ randomised controlled trial, $T 2 D$ type 2 diabetes

We did not apply meta-analytic methods to the identified studies because of axiomatic breaches in principles of meta-analysis (too few studies exploring a particular outcome, significant diversity in study design, confounder adjustment and analysis methods, multiple effectiveness estimates derived from same data source giving rise to possible double counting, and studies examining different GLP1RA agents; these breaches either rendered the effectiveness estimates uncombinable or would lead to a false illusion of precision) [11]. In addition there were too few studies for each outcome to provide funnel plots to assess the risk of publication bias (Cochrane Library recommends more than 10 studies) [12]. Thus we present a narrative systematic review.

This article is based on previously conducted studies and does not contain any new studies with human participants or animals performed by any of the authors.

\section{RESULTS}

Figure 1 shows a flow diagram of the studies included at each stage of applying our inclusion and exclusion criteria. The search results and the reviewers' inclusion and exclusion decisions are available on request. We present a synopsis of each study by outcome in Tables $2,3,4,5,6$, 7,8 and 9. Further information on the included studies are presented in Tables S5, S7, S8, S9, S10, S11, S12 and S13.

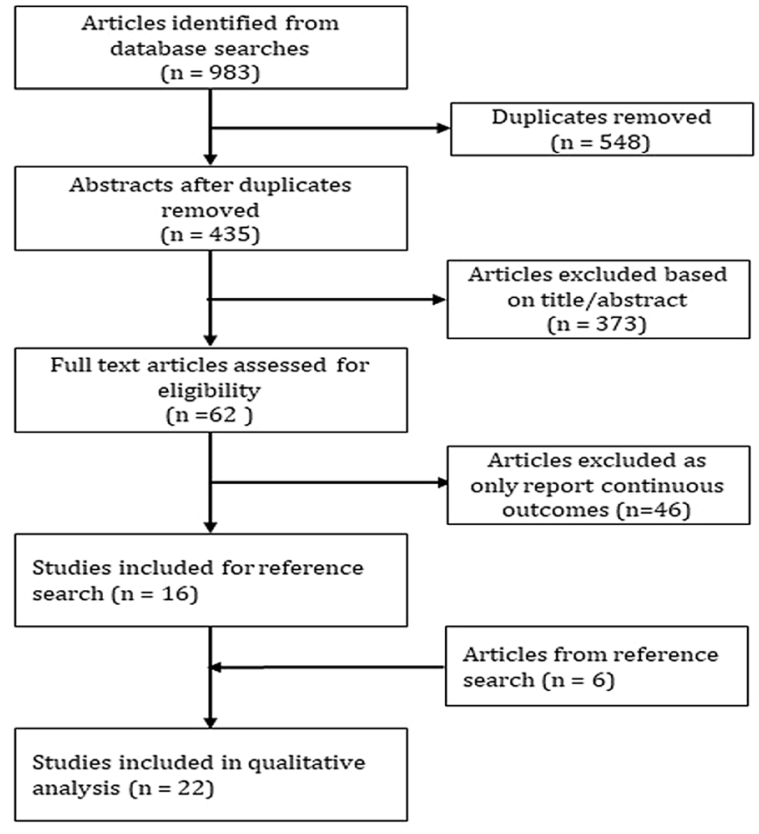

Fig. 1 PRISMA diagram

We found 22 studies in total of which 12 were commercially funded [13-25] and 10 were funded by academic/non-profit/government organisations [26-35]. Six studies were identified through reference searching. The total number of participants in the studies was 200,148 with follow-up of 396,457 person years (PYs).

Table S3 reports the quality score agreed by two independent reviewers (TC and JT). The 
Table 2 Cardiovascular disease

\section{Exposure Study synopsis}

GLP1RA class

2.1 A small 2016 American cohort study examined patients exposed to GLP1RA class agents (100\% on background metformin) versus insulin, SUs and DPP4Is head-to-head on CVD (composite of MI, UA, stroke and coronary revascularisation) [32]. A neutral association with CV events was observed comparing GLP1RAs head-to-head to insulin, SUs or DPP4Is, consistent according to baseline HbA1c (Tables S5.4 and S6). The CIs overall were narrow. This associated signal of neutral effect remained consistent in subgroup analysis (ontreatment and primary/secondary CVD prevention)

2.2 A small 2018 British cohort study assessed the effect of adding a GLP1RA class agent or an OAD to those already taking insulin and overweight on MACE, its components, a composite of the non-fatal MACE components and HF [26]. For MACE, there was an associated reduction for the GLP1RA class overall with narrow CIs (Table S5.5), with a reduced association also described for liraglutide, but no association for exenatide. CIs for the individual agents were wider than for the class overall. For the composite of the nonfatal components of MACE, there was no association for both the class and the individual agents, but with wide CIs (primary CVD prevention only). For the composite component of stroke, there no association for exenatide, liraglutide or for the class but with wide CIs in all three comparisons. For the MI composite component, there was no association in all three comparisons but with wide CIs. For HF, there was no association for all three comparisons, again with wide CIs (primary CVD prevention only). These associations were independent of baseline HbA1c (DNS). The associated reduction in MACE was driven by a reduction in ACM for the class overall and for the individual agents (Table S7.3)

2.3 A medium-sized 2017 British open cohort study explored GLP1RA class exposure vs. non-exposure (similar to OAD) [34]. As a secondary outcome (primary outcome was ACM, Table S7.1), in a population at low-risk of CVD, GLP1RA class exposure revealed a neutral association on incident CVD (Table S5.7). The CIs were narrow

Exenatide

2.4 A large 2011 American cohort study examined the effectiveness of exenatide BD exposure on incident CVD composite (MI, ischaemic stroke or coronary revascularisation) and CVD-related hospitalisation compared to no exenatide exposure in those already on other antihyperglycaemic drugs and without a CVD history in the preceding 9 months [13]. There was an associated reduction in the HR with exenatide exposure compared to no exposure in the PS-weighted, PS-stratified and the ITT analyses for CVD composite and for hospitalisation for CVD events. The CIs were all narrow. Authors observed lipid levels, BP, obesity and evidence of prior CVD were greater in those exposed to exenatide than those who were not, thus the exenatide-exposed would be at elevated baseline risk of CVD, lending support to exenatide being associated with a reduction in CVD (Table S5.1) 
Table 2 continued

Exposure Study synopsis

2.5 A medium-sized 2015 American cohort study compared the risk of CV outcomes in people exposed to exenatide $\mathrm{BD}$ versus insulin [33]. In the whole population, exenatide exposure was associated with a reduced risk of HHF, stroke and a composite of MI/stroke but not for MI alone, where there was no association (Table S5.2). The CIs were narrow for the MI and stroke composite and for HF, but wider for the individual composite components. Subgroup analyses in those without pre-existing CVD and without pre-existing CVD or CKD reported the same findings as the main analysis but also reported an associated reduction in MI for both. Those with higher baseline HbAlc had an associated greater reduction in HHF and MI or stroke with exenatide exposure than the unexposed (DNS)

2.6 A small 2015 American cohort study investigated primary prevention for time-to-first-HHF and all-cause hospitalisation for those exposed to exenatide versus those on OADs [35]. Exenatide exposure was associated no effect in all-cause hospitalisation, with wide CIs (Table S5.3), but there were insufficient cases to allow for assessment of HHF (DNS)

Liraglutide

2.7 A medium-sized 2019 Scandinavian cohort study (100\% on background metformin) compared liraglutide vs. DPP4I exposure on MACE [23]. Liraglutide was associated with a reduction in MACE (narrow CIs). This was driven by an associated reduction in the $\mathrm{CV}$ death composite component but not for MI or stroke. There was no association for HF and also for an expanded CV composite (MACE, other IHD, coronary revascularisation and PAD). The reduced association for MACE was greater for the secondary CVD prevention subgroup than the population overall, but attenuated to a neutral association in the primary prevention subgroup when analysed separately (Table S5.6). Subgroup analysis suggests a greater associated benefit in women, those $\geq 65$ years old and those on baseline insulin (DNS). For MACE, the associations remained stable in sensitivity analysis (on-treatment, additionally adjusted and imputed missing data, DNS)

$A C M$ all-cause mortality, $B D$ twice daily, $B P$ blood pressure, $C I$ confidence interval, $C K D$ chronic kidney disease, $C V D$ cardiovascular disease, DNS data not shown, DPP4I dipeptidyl peptidase 4 inhibitor, GLPIRA glucagon-like peptide 1 receptor agonist, $H b A 1 c$ glucated haemoglobin, $H F$ heart failure, $H H F$ hospitalised heart failure, $I H D$ ischaemic heart disease, ITT intention-to-treat, $M A C E$ major adverse cardiovascular events, $M I$ myocardial infarction, $O A D$ oral antidiabetic drug, $P A D$ peripheral artery disease, $P S$ propensity score, $S U$ sulfonylurea, $U A$ unstable angina

scores ranged from 12 to 22 (the maximum achievable score would have been $29 / 31$ as none of the studies was randomised). None of the study designs as described appeared to have been affected by immortal time bias arising from an inappropriate start date to follow-up.

The only individual agents investigated were exenatide or liraglutide, other agents were included in GLP1RA class studies but without information being provided on individual agent effectiveness or safety estimates.

\section{Cardiovascular Disease}

GLP1RA class: three studies explored the effect of the GLP1RA class overall on CVD with two finding no association and one finding an associated reduction in major adverse cardiovascular event (MACE) [26, 32, 34]. Specifically, when compared to other antihyperglycaemic agent (AHAs, including injectable therapies) head-to-head, one study (where the class comprised $72 \%$ exenatide, $29 \%$ liraglutide; $9-13 \%$ had established CVD, and $100 \%$ were on 
Table 3 Mortality

Exposure Study synopsis

GLP1RA class

3.1 A medium-sized 2017 British open cohort study explored the effects of GLP1RA class exposure vs. nonexposure on ACM [34]. Exposure was associated with reduced IRRs for ACM; subgroup analysis by agent also showed reduced associations for both liraglutide and exenatide when analysed separately. GLP1RA class association remained in the low risk of CVD subgroup (Table S7.1). All CIs were narrow. Subgroup analysis further suggests that the association for reduced ACM remained stable irrespective of baseline BMI, CVD and $\mathrm{BP}$, but there was a greater associated benefit in those with more DM complications and also with increasing age (DNS)

3.2 A small 2016 American cohort study (100\% on background metformin) explored ACM in those exposed to the GLP1RA class vs. insulin, SUs and DPP4Is head-to-head [32]. There was a neutral association with ACM in all three pairwise comparisons, but the CIs were wide (Tables S6 and S7.5)

3.3 A small 2018 British cohort study examined liraglutide or exenatide plus insulin vs. OAD plus insulin's effect on ACM (100\% on insulin) [26]. This showed an associated reduction in ACM with liraglutide, exenatide and the GLP1RA class exposure (Table S7.3), independently of HbAlc reduction (DNS). The CIs were wide, wider still for the individual drug comparisons. The associated rate of ACM remained lower following adjustment for age, duration of DM, insulin use, gender, socioeconomic status, alcohol use, eGFR, lipid profile and hypoglycaemia (DNS)

Exenatide

3.4 A small 2015 American cohort study explored the effect of exenatide exposure vs. non-exposure on ACM [35]. There was no association in ACM with exenatide exposure, but with very wide CIs (Table S7.2). There was no associated difference in CVD or HF mortality between the groups (DNS)

Liraglutide

3.5 A large 2019 Scandinavian cohort study (100\% on background metformin) explored liraglutide exposure's effect on ACM and CV mortality vs. DPP4I [23]. This study showed an associated reduction in both ACM and CV death in those exposed to liraglutide (Table S7.4). The CIs were narrow

$A C M$ all-cause mortality, $B M I$ body mass index, $B P$ blood pressure, $C I$ confidence interval, $D M$ diabetes mellitus, $D N S$ data not shown, DPP4I dipeptidyl peptidase 4 inhibitor, eGFR estimated glomerular filtration rate, GLPIRA glucagon-like peptide 1 receptor agonist, $H b A I c$ glucated haemoglobin, $H F$ heart failure, IRR incidence rate ratio, $O A D$ oral antidiabetic drug, $S U$ sulfonylurea

background metformin) showed no association for MACE (and its components; Tables 2.1, S5.4, S6), irrespective of baseline HbA1c and consistent in subgroup analysis [32]. Versus oral antidiabetic drug (OADs, excluding injectable therapies) (in those already exposed to insulin and overweight; exenatide $72 \%$, liraglutide 28\%, 100\% background insulin, 20\% baseline CVD; Tables 2.2 and S5.5), another study showed an associated reduction in MACE (driven by a mortality reduction). In the same study, for a composite of non-fatal MACE components and for hospitalised heart failure (HHF) (primary CVD prevention only) there was no association [26]. Another study of a population at low risk of CVD suggested that GLP1RA exposure was associated with no effect on incident CVD compared to people not exposed (Tables 2.3 and S5.7) [34]. 
Table 4 Pancreatic outcomes

\section{Exposure Study synopsis}

Exenatide

4.1 A large 2010 American cohort study assessed the risk of AP in those exposed to exenatide BD compared to those not exposed to incretin therapy and also to people without DM [28]. All participants did not have claims for AP in the preceding 6 months. The CIs were wide. Although a diagnosis of DM was associated with an increased risk for AP, compared to OADs, exenatide exposure was associated with a neutral effect on AP (Table S8.1)

4.2 A large 2011 American cohort study explored the association of exenatide BD vs. those using OAD with AP (using an adjudicated definition) in people without prior pancreatic disease [15]. There was a reduced association in the PS-adjusted analysis with current use of exenatide; a neutral association with recent use of exenatide compared to OAD; and an increased association with past exenatide use. All of the CIs were wide. The nested (within cohort) case-control study showed the same effect for the current use (DNS) and recent use (DNS), but for past use it showed a neutral association (Table S8.2)

4.3 A large 2010 American cohort study explored the association of exenatide BD initiation on time-to-first-AP vs. OAD [25]. There was a neutral association for AP with exenatide in the main IPTW analysis, and also in the crude, ITT and PS-stratified analysis (Table S8.3). Current and recent exenatide exposure also showed a neutral association, whereas past exposure showed a reduced association for AP. The CIs were narrow for current and past exposure, but wide for recent exposure

4.4 A large 2012 American cohort study explored the association of exenatide BD vs. non-users on AP and PC [22]. Furthermore, a quasi-randomised sensitivity analysis using out-of-pocket expenses was also undertaken. The logistic regression and quasi-randomised analyses showed a neutral association of exenatide with both AP and PC (Table S8.4). The CIs for AP were narrow, as was the CI in the quasi-randomised PC analysis; CIs were wide in the main cohort PC analysis

4.5 A small 2014 American SCCS study explored exenatide BD use (controlling for time-invariant confounders) on AP [30]. The study found that exenatide exposure was associated with a neutral effect on the IDR of AP in each time window and in the modified analyses (Table S8.5). The CIs were wide

4.6 A large 2019 American cohort (and nested case-control) study examined PC incidence in patients initiated on exenatide $\mathrm{BD}$ or $\mathrm{QW}$ vs. OADs $[14,19]$. The study showed no association for exenatide exposure on PC in the main cohort, the cumulative response and the nested case-control analysis (Table S8.8). The CIs were wide

Liraglutide

4.7 A 2014 American cohort study compared liraglutide vs. OADs for association with AP and PC (study size not ascertainable) [18]. Liraglutide exposure was associated with a neutral effect for AP and no association for PC. This signal was consistent between both the ITT and on-treatment analysis (Table S8.6). All CIs were wide 
Table 4 continued

\section{Exposure Study synopsis}

4.8

A medium-sized 2019 American cohort study compared adjudicated AP and PC outcomes from individuals exposed to liraglutide vs. OAD [16]. ITT and TOD analyses were performed which showed a neutral effect of liraglutide for AP and PC overall (Table S8.7). For liraglutide, the association for AP and PC remained neutral in each of the head-to-head drug comparisons (vs. DPP4I, SUs and TZDs) when repeated individually. CIs were wide

$A P$ acute pancreatitis, $B D$ twice daily, $C I$ confidence interval, $D M$ diabetes mellitus, $D P P 4 I$ dipeptidyl peptidase 4 inhibitor, $I D R$ incidence density ratio, $I P T W$ inverse probability of treatment weighting, ITT intention-to-treat, $O A D$ oral antidiabetic drug, $P C$ pancreatic cancer, $P S$ propensity score, $Q W$ once weekly, $S C C S$ self-controlled case series, $S U$ sulfonylurea, $T O D$ time-on-drug, $T Z D$ thiazolidinedione

Table 5 Thyroid cancer

\section{Exposure Study synopsis}

Exenatide

5.1 A large 2019 American cohort (and nested case-control) study of exenatide exposure vs. OAD explored the association of exenatide with TC $[14,19]$. There was no association for TC in the main cohort and the cumulative response (both with wide CIs) or the nested case-control analysis (with narrower CIs; Table S9.1)

$C I$ confidence interval, $O A D$ oral antidiabetic drug, $T C$ thyroid cancer

Table 6 Renal outcomes

\section{Exposure Study synopsis}

Exenatide

6.1 A medium-sized 2012 American cohort study examined the risk of incident ARF associated with exenatide exposure vs. OADs (excluding sitagliptin) in patients without baseline ARF, ESRD, renal dialysis or renal transplant (baseline CKD 1.9\%) [21]. There was no association in the time-to-renal-failure Kaplan-Meier analysis for exenatide exposure with ARF (Table S10.1). When the analysis was restricted to patients with high risk of ARF, when the data was censored at 180 days and when a modified diabetic control group was used there remained no association of exenatide exposure with ARF (DNS). The CIs were narrow

$A R F$ acute renal failure, $C I$ confidence interval, $C K D$ chronic kidney disease, $D N S$ data not shown, ESRD end-stage renal disease, $O A D$ oral antidiabetic drug

Exenatide: we found four studies that reported a CVD-related outcome for exenatide alone. Three reported a reduction in CVD associated with exenatide, and one reported no association $[3,13,33,35]$.
Compared to OADs: one study showed an associated reduction with exenatide exposure for a myocardial infarction (MI), stroke and coronary revascularisation composite and also CVD-related hospitalisation (where lipid levels, 
Table 7 Diabetic retinopathy

\section{Exposure Study synopsis}

\section{GLP1RA class}

7.1 A medium-sized 2018 British cohort study investigated the association of incident DR (in those without prevalent DR) in GLP1RA class exposure vs. $>2$ OADs [27]. The study included negative (DPP4I) and positive (insulin) controls (DNS). Exenatide exposure was associated with a reduced effect on DR in the main cohort and $>12$ months use; a neutral association at $<6$ months use; and an increased association for the $>6$ to $\leq 12$-month time window (Table S11.1). The CIs were narrow. There was no associated effect modification by duration of DM treatment and HbA1c levels, but GLP1RA-associated DR was increased among patients with arterial hypertension and on ACEI (DNS)

$A C E I$ angiotensin-converting enzyme inhibitor, $C I$ confidence interval, $D M$ diabetes mellitus, $D N S$ data not shown, DPP4I dipeptidyl peptidase 4 inhibitor, $D R$ diabetic retinopathy, GLPIRA glucagon-like peptide 1 receptor agonist, HbAlc glucated haemoglobin, $O A D$ oral antidiabetic drug

Table 8 Breast cancer

\section{Exposure Study synopsis}

GLP1RA class

8.1 A small 2016 British cohort study compared those taking GLP1RA class agents vs. DPP4Is on the risk of incident breast cancer [29]. Detection bias was handled in sensitivity analysis by varying lag time windows, censoring in situ breast cancer, performing an IPTW analysis and stratifying by mammography screening. Overall, there was a neutral association of GLP1RA class on breast cancer (and also for exenatide and liraglutide alone, when analysed separately). The dose-response analysis was associated with a transient increase in the breast cancer risk of those in the $>3$ to $\leq 4$-year exposure window, which attenuated in longer exposure (Table S12.1). Sensitivity analyses yielded similar results to the primary analysis, except that those with no mammography screening had an association with increased breast cancer risk (DNS). All of the CIs were wide

Liraglutide

8.2 A large 2018 American cohort study compared individuals taking liraglutide vs. OADs on the risk of developing adjudicated incident breast cancer [17]. ITT and cumulative TOD analyses were performed. Detection bias was handled comparing mammography frequency between the groups. Liraglutide exposure was associated with a neutral risk of breast cancer in both the ITT (for all comparator agents) and also in the cumulative TOD analyses (Table S12.2). The CIs were narrow, except the recent TOD which was wide

$C I$ confidence interval, DNS data not shown, DPP4I dipeptidyl peptidase 4 inhibitor, GLPIRA glucagon-like peptide 1 receptor agonist, $I P T W$ inverse probability of treatment weighting, ITT intention-to-treat, $O A D$ oral antidiabetic drug, TOD time-on-drug

obesity and prior CVD rates were higher in the exenatide-exposed; Tables 2.4 and S5.1) [13]. Another showed no association in all-cause hospitalisation, but featured insufficient cases to allow for assessment on HHF (Tables 2.6 and S5.3) $[3,35]$. 
Table 9 Hypoglycaemia

Exposure Study synopsis

GLP1RA class

9.1 A small 2018 American cohort study investigated rates of hypoglycaemic events in those exposed to GLP1RA class agents (comparing early GLP1RA treatment intensification, delayed or no treatment intensification vs. insulin) [24]. Treatment intensification (early or delayed) was not associated with a change in risk of hypoglycaemia compared to insulin (Table S13.2). The CI widths varied depending on comparison

Exenatide

9.2 A small 2017 American cohort study investigated the association of exenatide QW on hypoglycaemia vs. basal insulin in patients naïve to injectable therapy by ethnicity [31]. Exenatide exposure was associated with a neutral effect on the rates of hypoglycaemia events in all groups (Table S13.1). CIs were narrow for the white ethnicity but wide for the other ethnic groups. For background characteristics, see Table S14

9.3 A medium-sized British 2018 cohort study explored the association with severe hypoglycaemia in those previously naïve to any exenatide therapy vs. basal insulin \pm OAD [20]. No association for exenatide on severe hypoglycaemia was observed, although the absolute rates of hypoglycaemia were higher in the insulinexposed group compared to exenatide (DNS). No effect estimates or CIs reported

$C I$ confidence interval, DNS data not shown, GLPIRA glucagon-like peptide 1 receptor agonist, OAD oral antidiabetic drug, $T O D$ time-on-drug, $Q W$ once weekly

Compared to insulin (which is known to increase the risk of CVD [36]), exenatide exposure was associated with a reduction in HHF, stroke and an $\mathrm{MI} /$ stroke composite but not MI alone (Tables 2.5 and S5.2); there was an associated reduction in all outcomes when those without pre-existing CVD or CVD/chronic kidney disease (CKD) were analysed separately [33]. Those with higher baseline HbA1c had a greater associated reduction in HHF alone and MI/ stroke composite. A final study for exenatide showed no association towards reduction in MACE. For primary CVD prevention only, there was no association for the non-fatal CVD composite, MI, HHF and stroke (independent of baseline HbA1c; data not shown (DNS)) (Tables 2.2 and S5.5) [26].

Liraglutide: two studies reported the effect of liraglutide on CVD [23, 26]. Both showed reductions in MACE in the population overall (driven by a mortality reduction), in populations with less established CVD - 20\% [26] and $16 \%$ [23] vs. $81 \%$ in Liraglutide Effect and Action in Diabetes: Evaluation of cardiovascular outcome Results (LEADER) [6] and similar levels of baseline glycaemic control [23, 26]. Against dipeptidyl peptidase 4 inhibitors (DPP4i), one study showed a reduction in MACE overall and in secondary, but not primary, CVD prevention (Tables 2.7 and S5.6). Women, those aged 65 years or more, and those on baseline insulin benefitted most from this associated reduction in subgroup analysis (DNS) [23]. Versus insulin, the other study showed an associated reduction in MACE but no association for MI, stroke, MI/ stroke composite and for HHF, independent of baseline HbA1c (Tables 2.2 and S5.5; MI/stroke/ HHF explored primary prevention only, DNS) [26].

\section{Mortality}

For GLP1RA class overall (vs. unexposed, i.e. usual care) exposure was associated with an allcause mortality (ACM) reduction irrespective of baseline body mass index (BMI), CVD status and BP (Tables 3.1 and S7.1) but a greater associated benefit in those with more diabetes mellitus (DM) complications and in older people (DNS) [34]. Against insulin, sulfonylurea (SUs) and 
DPP4Is head-to-head, there was no association with ACM in all comparisons (Tables 3.2 and S7.5) [32]. Against OADs + insulin, there was an associated reduction for ACM (Tables 3.3 and S7.3) [26].

For exenatide, two studies were associated with a reduction in ACM $[26,34]$, and one showed no association [35]. Versus non-exposure, the associated reduction in ACM was irrespective of baseline BMI, CVD status and BP (Tables 3.1 and S7.1) but a greater associated benefit was seen in those with more DM complications and in older people (DNS) [34]. Versus OADs and insulin, there was an associated reduction in ACM (Tables 3.3 and S7.3) [26]. Finally, versus the unexposed, there was no association with ACM (Tables 3.4 and S7.2) [35].

For liraglutide, all three studies were associated with a reduced risk of ACM, two vs. OAD $[26,34]$ and one vs. DPP4I [23] (Tables 3.1 [34], 3.3 [26], 3.5 [23], and S7.1 [34], S7.3 [26], S7.4 [23]). In one study there was also an associated reduction in CV death (Tables 3.5 and S7.4) [23]. For another, this was irrespective of baseline BMI, CVD status and BP but a greater associated benefit was shown in those with more DM complications and in older people (DNS) [34].

\section{Pancreatic Outcomes}

We found eight studies reporting the association of GLP1RAs with pancreatic outcomes, seven vs. OADs/usual care $[14,16,18,19,22,25,28]$ and one as an selfcontrolled case series (SCCS) [30].

\section{Acute Pancreatitis}

We found seven studies that explored acute pancreatitis (AP) following GLP1RA exposure $[15,16,18,22,25,28,30]$, five for exenatide $[15,22,25,28,30]$ and two for liraglutide [16, 18] (Fig. 2).

For exenatide, four studies showed no association with AP following exposure (Tables 4.1 [28] and S8.1, 4.3 [25] and S8.3, 4.4 [22] and S8.4, 4.5 [30] and S8.5); in one study when 'outof-pocket' expenses were used as a 'quasi-randomised' variable, there remained no association for AP [22]. Another study suggested an associated reduction in AP (Tables 4.2 and S8.2) [15]. When time post-exposure was considered, the same study showed an associated increase in AP with past exenatide use [15], but a different study suggested an associated reduction for past exposure (Tables 4.3 and S8.3) [25]. In the case of an SCCS, although throughout the study the associated effect of exenatide on both recurrent (DNS) and incident AP increased over time following exposure (as did both bounds of the $95 \%$ CIs), the effect estimates did not reach statistical significance (Tables 4.5 and S8.5) [30].

For liraglutide, two studies showed no associated with AP (Tables 4.7 [16] and S8.6, 4.8 [18] and S8.7). In one of these studies, there remained no association for $\mathrm{AP}$ in both the intention-to-treat (ITT) and on-treatment analysis [18].

\section{Pancreatic Cancer}

We found four studies that explored pancreatic cancer incidence following GLP1RA exposure, two for exenatide $[14,19,22]$ and two for liraglutide [16, 18] (Fig. 3).

For exenatide, two studies showed no association following exposure (Tables 4.4, S8.4 and Tables 4.6, S8.8, the latter in the main cohort, cumulative response and nested case-control analyses) [14, 19, 22].

For liraglutide, two studies showed no association in all analyses (Tables 4.7 [18] and S8.6, 4.8 [16] and S8.7). In one of these there was no association for pancreatic cancer (PC) in both the ITT and on-treatment analysis in the 2014 study [18].

\section{Thyroid Cancer}

No study we found explored medullary thyroid cancer (MTC) (the specific cancer type putatively associated with GLP1RA exposure, Table 1). The only study identified reported no association with the risk of TC (which included, but was not limited to, MTC) with exenatide exposure, vs. OAD, in the main cohort and the cumulative dose-response analysis, nor the 


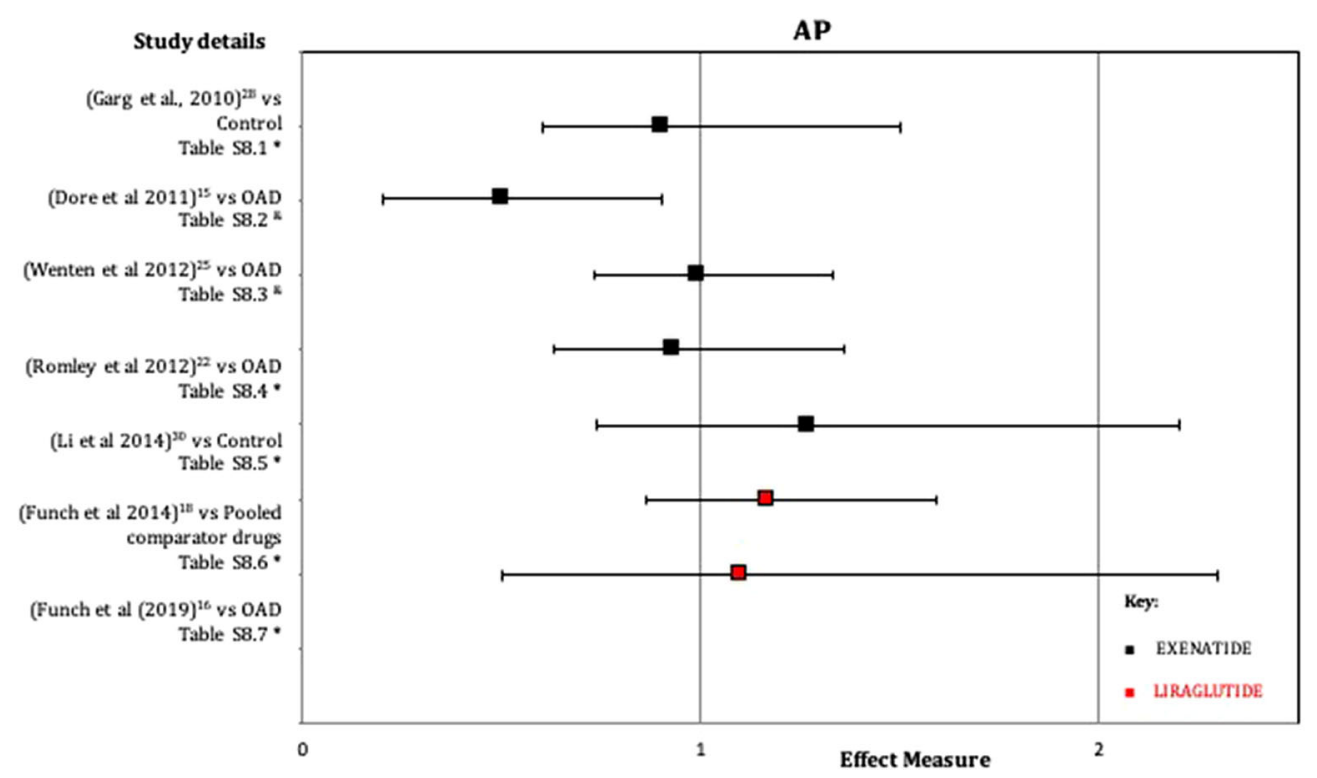

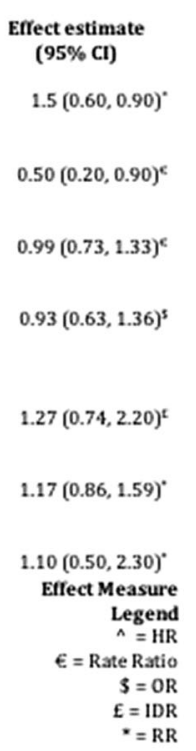

Fig. 2 Acute pancreatitis forest plot

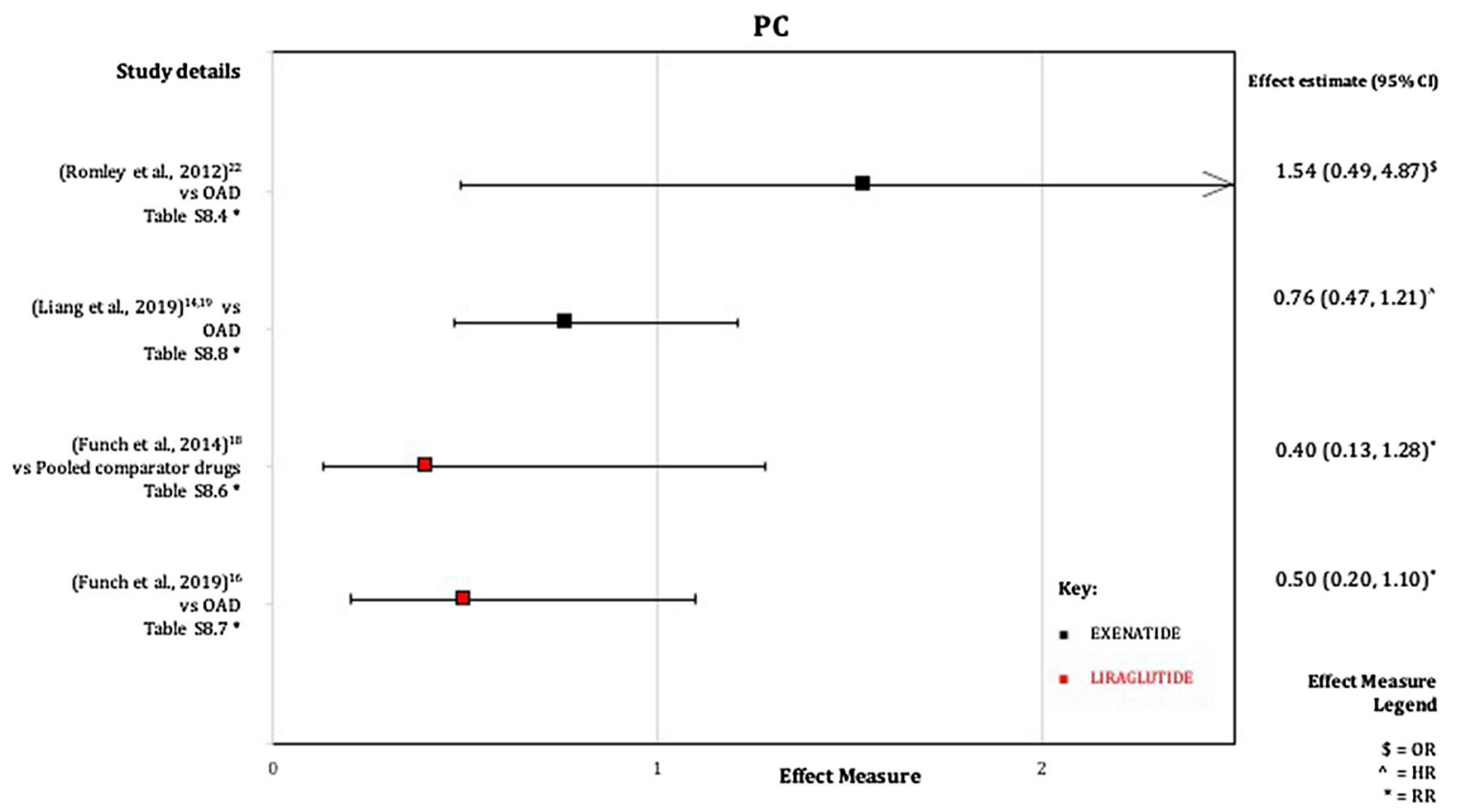

Fig. 3 Pancreatic cancer forest plot

nested case control study (Tables 5.1 and S9.1) Renal Outcomes $[14,19]$.

Only one study described renal outcomes, which suggests that exenatide exposure (vs. 
sitagliptin) was not associated with an increased time-to-renal-failure or acute renal failure (ARF), in which the majority of participants did not have baseline CKD (Tables 6.1 and S10.1) [21].

\section{Diabetic Retinopathy}

The one study we identified showed no association of GLP1RA class (vs. more than two OADs) on diabetic retinopathy (DR) incidence in those without pre-existing eye disease (Tables 7.1 and S11.1) [27]. No effect modification was detected by duration of DM treatment or baseline HbA1c but there was an increased risk of DR in those with hypertension and in those already on an angiotensin-converting enzyme inhibitor (ACEI).

\section{Breast Cancer}

The two studies we identified assessing the effect of GLP1RA exposure on breast cancer (vs. DPP4I [29], Tables 8.1 and S12.1 and vs. OAD [17], Tables 8.2 and S12.2) showed that both the GLP1RA class overall and the specific agents liraglutide and exenatide were associated with a neutral effect on risk of incident breast cancer, consistent in the duration response and ITT analyses in both studies overall [17, 29], apart from an associated transient increased risk at more than 3.1 years to less than 4 years in one [29].

\section{Hypoglycaemia}

We found three studies exploring rates of severe hypoglycaemia, two investigating exenatide exposure (Tables 9.2 [31] and S13.1, and 9.3 [20]) and one the GLP1RA class overall (Tables 9.1 [24] and S13.2). All studies used an insulin comparator group (as opposed to OAD) and showed no association with hypoglycaemia risk $[20,24,31]$.

\section{DISCUSSION}

\section{Summary of Key Findings}

For context, Table 1 gives a summary of the RCT (or other source of) evidence for the outcomes described in this review. Notably, of the individual GLP1RA agents, only studies exploring exenatide and liraglutide were found (because these have been available longest). Consequently, there is a need to undertake large observational studies of newer GLP1RA drugs (dulaglutide, semaglutide and lixisenatide). This discussion is, consequently, mainly limited to the GLP1RA class as a whole, exenatide and liraglutide.

\section{Effectiveness}

\section{Cardiovascular Disease}

For the GLP1RA class, studies either showed no association $[32,34]$ or an associated reduction [26] with CVD. These studies appear to confirm CVD safety for the GLP1RA class overall.

For exenatide, two studies reported an associated reduction for $\operatorname{CVD}[13,33]$ and two studies reported no association [26, 35]. While these data confirm CVD safety, given that the cardiovascular outcome trial (CVOT) for exenatide did not show a reduction in CVD, exenatide should not be prescribed for the purposes of reducing CVD in people with T2D.

For liraglutide, two studies demonstrated an associated reduction for CVD [23, 26]. These findings first confirm CVD safety, but since liraglutide reduced MACE in the CVOT, they also confirm and extend these findings to those living with T2D but with a broader CV risk profile than those at elevated $\mathrm{CV}$ risk included in the CVOT for enrichment purposes.

\section{Mortality}

For the GLP1RA class, two studies showed an associated reduction in ACM $[26,34]$ and one showed no association [32]. No studies explored CV death.

For exenatide two studies were associated with a reduction in ACM [26, 34] and another showed no association [35]. No studies explored 
CV death. EXenatide Study of Cardiovascular Event Lowering Trial (EXSCEL) showed nominally significant reduction in ACM (but not for CV death) [3]; for ACM these studies appear to suggest that exenatide may be associated with mortality postponement in a broad population and certainly does not appear to increase morality risk.

For liraglutide, all three studies demonstrated an associated reduction for ACM $[23,26,34]$ and one study also showed a reduction in CV death [23]. LEADER showed a reduction in ACM and $\mathrm{CV}$ death [6]; for both these outcomes the studies we found appear to confirm that liraglutide may be associated with a postponement of both ACM and CV death in these broad populations.

\section{Safety}

The current regulatory position for GLP1RA safety is as follows. The European Medicines Agency (EMA) has issued a class-wide warning for AP [37] and specifically for semaglutide, for worsening of DR [38]. For semaglutide only, the US Food and Drug Administration (FDA) warns of worsening of DR, particularly in those already treated with insulin [39]. The FDA has issued class-wide warnings for AP and PC [40], for MTC [39], for ARF [39] and recently for hypoglycaemia in the context of co-prescription with Sus [39]. The Medicines and Healthcare products Regulatory Agency (MHRA) has recently warned about diabetic ketoacidosis (DKA) in the context of rapid insulin dose-reduction following GLP1RA initiation [41]. Not all of the agents/outcomes are addressed by studies included in this review.

\section{Acute Pancreatitis}

Seven studies explored this outcome $[15,16,18,22,25,28,30]$. For exenatide, four studies suggest no association [22, 25, 28, 30] and one an associated reduction [15]. For liraglutide all studies reported no association $[16,18]$. In summary, the studies we found do not suggest that GLP1RAs (as a class or individual agent) are associated with AP.

\section{Pancreatic Cancer}

Four studies explored this outcome $[16,18,19,22]$. For exenatide there was no association $[19,22]$. For liraglutide both studies suggest no association for this outcome $[16,18]$. Overall, these findings provide reassurance that neither the class overall nor the individual agents examined appear to be associated with PC over longer-term exposure.

\section{Thyroid Cancer}

One study explored this outcome [14, 19], which suggests no association for exenatide exposure for this outcome (for TCs in general and not MTC specifically). It is difficult to draw conclusions from a single study and this outcome should be investigated further.

\section{Renal Outcomes}

One study explored the association of exenatide with ARF [21]. Exposure did not appear to be associated with time-to-ARF and appears safe from the renal perspective. None of the outcomes trials explored renal events as a primary outcome (and were not reported in EXSCEL), although a secondary analysis of LEADER suggests that liraglutide exposure caused a reduction in the renal composite outcome (newonset persistent microalbuminuria, persistent doubling of the serum creatinine level, ESRD, or death due to renal disease) driven mainly by a reduction in persistent microalbuminuria $[3,5,42]$. However, exenatide is renally excreted and should not be used in those with pre-existing renal impairment [43].

\section{Diabetic Retinopathy}

One study explored GLP1RA class exposure on DR and was associated with a neutral effect on this outcome (except a transient increase in risk at 6-12 months) [27]. It is difficult to draw conclusions from a single study and this outcome ought to be investigated further. In those with pre-existing eye disease caution should be employed and, in particular, slow titration should be considered to avoid rapid correction of hyperglycaemia which may precipitate DR. 


\section{Breast Cancer}

Two studies explored this outcome [17, 29]. One study showed no association with breast cancer for the GLP1RA class overall, for exenatide and for liraglutide (except a transient increased in risk at 3-4 years) [29]. The other study showed no association for liraglutide [17]. These data appear to suggest that GLP1RA agents are not associated with an increased risk for this outcome.

\section{Hypoglycaemia}

Three studies explored this outcome $[20,24,31]$. For the GLP1RA class and for exenatide, the studies showed no association of exposure with hypoglycaemia (compared to insulin). GLP1RAs alone do not appear to increase the risk of hypoglycaemia, but may increase hypoglycaemia risk when co-prescribed with other drugs known to cause low blood glucose [39]. Given that the studies identified were compared directly to insulin use (and not co-prescription with insulin), these data do not shed light on this important question.

\section{Strengths and Limitations}

To our knowledge, this is the first systematic review to assess population-based observational studies looking at the effectiveness and safety of GLP1RA agents in T2D. We searched a large number of studies and involved a University of Edinburgh informationist to use appropriate search terms to ensure we captured the largest possible number of studies. We present data from many different countries and diverse populations of people living with T2D and the quality of the included papers is reported.

Importantly, some outcomes, particularly pancreatic disease, were assessed by combining GLP1RA and DPP4I exposure by assuming a common 'incretin' pathway leading to these pathologies (e.g. Singh et al. [44]). These studies, where it was not possible to discern an effect estimate for a GLP1RA agent alone, were prospectively excluded from this review (Table S2).

A major challenge was the variety of composite endpoints in the studies identified, particularly for CVD, making direct comparisons difficult. While composite primary endpoints is useful for increasing the power of a study to detect change (by increasing the events counted towards the primary endpoint) it would be helpful if future studies emulated the composite outcomes reported in the cardiovascular outcomes trials. This would be useful for comparing identified studies to each other and also to RCT data.

CVOTs have shown that liraglutide, subcutaneously administered semaglutide and dulaglutide reduce MACE $[2,6,7]$. Given that the last two agents have been licensed more recently, we did not identify population-based studies exploring these agents. It will be important to assess these agents' effects on CVD in day-to-day clinical practice once sufficient person-time exposure has accumulated to allow for such analyses.

We deliberately excluded disproportionality reporting ratio studies based on interrogating adverse event reporting databases because of the high risk of bias, especially once an adverse event becomes known. However, gastroesophageal reflux-like symptoms [45] and intestinal obstruction [46] both emerged as potential harms from these studies and should be investigated further.

As with all observational data exploring treatment effectiveness, the studies included may be subject to unmeasured confounding and bias, particularly measurement/misclassification bias. Using population-based studies reduces the effect of selection bias but observational pharmacoepidemiology is always subject to confounding-by-indication, although most of the included studies used modern methods of controlling for this (e.g. propensity score matching, PSM). It remains the case that despite adequate study design and analysis methods, the associations described might be entirely explained away, or even reversed, by bias and confounding, particularly unmeasured confounding.

Notwithstanding considerable effort on our part to identify eligible studies systematically (including the manual searching of references), it remains possible that we overlooked potentially suitable studies and we omitted non- 
English language publications as a result of resource issues; thus, our findings may be affected by publication bias, which we could not measure using a funnel plot because of insufficient numbers of studies. Although the search terms we employed should detect observational studies conforming to the Strengthening The Reporting of OBservational studies in Epidemiology (STROBE) items on publication title and abstract, studies not conforming would be missed (but non-conforming could be considered a marker of poor quality at any rate).

We did not use meta-analytic methods in this review because of axiomatic breaches of the methodology, including significant study diversity and possible double counting of outcome events.

\section{CONCLUSIONS}

This work provides broadly generalisable effectiveness estimates in more heterogeneous populations than those included in CVOTs. Our study confirms that GLP1RAs, either as a class or as individual agents, do not appear to be associated with an increased risk of $\mathrm{CV}$ events and are safe from this perspective. For liraglutide, our review confirms and extends trial findings that this agent appears to be associated with a reduction in $\mathrm{CV}$ events of similar direction and order of magnitude to the CVOT.

The included studies did not find evidence of previously reported safety outcomes, although some outcomes were only explored in a single study (or a small number of studies). GLP1RAs do not appear to be associated with pancreatic pathology, TC, worse renal outcomes (in those without baseline CKD), incident DR (in those without baseline DR), incident breast cancer and displayed a decreased or no association on the risk of hypoglycaemia (when compared to insulin/OAD respectively). Given the heterogeneity of the studies in terms of outcome definition, population, confounding control and analysis method, making generalised conclusions about these data is challenging.

These data will be of use to people living with $\mathrm{T} 2 \mathrm{D}$, their clinicians, medical regulators and guideline-writing organisations and should be updated as more data become available.

\section{ACKNOWLEDGEMENTS}

We would like to thank Ruth Jenkins at the University of Edinburgh Library. TC acknowledges the support of Diabetes UK ('Sir George Alberti' Clinical Research Training Fellowship 18/0005786). RMR acknowledges the support of the British Heart Foundation (RE/18/5/34216).

Funding. No funding or sponsorship was received for this study or publication of this article. The Rapid Service Fee was funded by the authors.

Authorship. All named authors meet the International Committee of Medical Journal Editors (ICMJE) criteria for authorship for this article, take responsibility for the integrity of the work as a whole, and have given their approval for this version to be published.

Authorship Contributions. TMC and JBT conceived, designed and executed this study. TAC provided critical manuscript revision and restructuring figure and table construction and approved the final version. SHW and RMR critically reviewed the draft manuscript and approved the final version. DJW provided manuscript review/editing, expert clinical pharmacology opinion and research supervision. HMC provided expert pharmacoepidemiology opinion and research supervision.

Disclosures. Thomas M. Caparrotta, Jack B. Templeton, Thomas A. Clay, Sarah H. Wild, Rebecca M. Reynolds, David J. Webb, Helen M. Colhoun report disclosures or no conflicts of interest.

Compliance with Ethics Guidelines. This article is based on previously conducted studies and does not contain any new studies with human participants or animals performed by any of the authors. 
Data Availability. All data generated or analysed during this study are included in this published article/as supplementary information files.

Open Access. This article is licensed under a Creative Commons Attribution-NonCommercial 4.0 International License, which permits any non-commercial use, sharing, adaptation, distribution and reproduction in any medium or format, as long as you give appropriate credit to the original author(s) and the source, provide a link to the Creative Commons licence, and indicate if changes were made. The images or other third party material in this article are included in the article's Creative Commons licence, unless indicated otherwise in a credit line to the material. If material is not included in the article's Creative Commons licence and your intended use is not permitted by statutory regulation or exceeds the permitted use, you will need to obtain permission directly from the copyright holder. To view a copy of this licence, visit http://creativecommons.org/licenses/by$\mathrm{nc} / 4.0 /$.

\section{REFERENCES}

1. Nauck MA, Meier JJ. Incretin hormones: their role in health and disease. Diabetes Obes Metab. 2018;20:5-21.

2. Gerstein HC, Colhoun HM, Dagenais GR, et al. Dulaglutide and cardiovascular outcomes in type 2 diabetes (REWIND): a double-blind, randomised placebo-controlled trial. Lancet. 2019;394:121-30.

3. Holman RR, Bethel MA, Mentz RJ, et al. Effects of once-weekly exenatide on cardiovascular outcomes in type 2 diabetes. N Engl J Med. 2017;377:1228-39.

4. Husain M, Birkenfeld AL, Donsmark M, et al. Oral semaglutide and cardiovascular outcomes in patients with type 2 diabetes. $\mathrm{N}$ Engl J Med. 2019;381:841-51.

5. Mann JFE, Ørsted DD, Brown-Frandsen $\mathrm{K}$, et al. Liraglutide and renal outcomes in type 2 diabetes. N Engl J Med. 2017;377:839-48.

6. Marso SP, Daniels GH, Brown-Frandsen K, et al. Liraglutide and cardiovascular outcomes in type 2 diabetes. N Engl J Med. 2016;375:311-22.
7. Marso SP, Bain SC, Consoli A, et al. Semaglutide and cardiovascular outcomes in patients with type 2 diabetes. N Engl J Med. 2016;375:1834-44.

8. Pfeffer MA, Claggett B, Diaz R, et al. Lixisenatide in patients with type 2 diabetes and acute coronary syndrome. N Engl J Med. 2015;373:2247-57.

9. Downs $\mathrm{SH}$, Black N. The feasibility of creating a checklist for the assessment of the methodological quality both of randomised and non-randomised studies of health care interventions. J Epidemiol Community Health. 1998;52:377-84.

10. Hootman JM, Driban JB, Sitler MR, Harris KP, Cattano NM. Reliability and validity of three quality rating instruments for systematic reviews of observational studies. Res Synth Methods. 2011;2:110-8.

11. Berlin JA, Crowe BJ, Xia HA, Evans SJW. The use of metaanalysis in pharmacoepidemiology. In: Strom BL, Kimmel SE, Hennessy S, editors. Pharmacoepidemiology. Chichester: Wiley; 2019. p. 897-947.

12. Cochrane Collaboration. 10.4.1 Funnel plots. https://handbook-5-1.cochrane.org/chapter_10/ 10_4_1_funnel_plots.htm. Accessed 26 Nov 2020.

13. Best JH, Hoogwerf BJ, Herman WH, et al. Risk of cardiovascular disease events in patients with type 2 diabetes prescribed the glucagon-like peptide 1 (GLP-1) receptor agonist exenatide twice daily or other glucose-lowering therapies: a retrospective analysis of the Lifelink database. Diabetes Care. 2011;34:90-5.

14. Liang C. Corrigendum: Exenatide use and incidence of pancreatic and thyroid cancer: a retrospective cohort study. Diabetes Obes Metab. 2020;22:1006-1006.

15. Dore DD, Bloomgren GL, Wenten M, et al. A cohort study of acute pancreatitis in relation to exenatide use. Diabetes Obes Metab. 2011;13:559-66.

16. Funch D, Mortimer K, Ziyadeh NJ, et al. Liraglutide use and evaluation of pancreatic outcomes in a US commercially insured population. Diabetes Obes Metab. 2019;21:1837-48.

17. Funch D, Mortimer $\mathrm{K}, \mathrm{Li} \mathrm{L}$, et al. Is there an association between liraglutide use and female breast cancer in a real-world setting? Diabetes Metab Syndr Obes. 2018;11:791-806.

18. Funch D, Gydesen H, Tornøe K, Major-Pedersen A, Chan KA. A prospective, claims-based assessment of the risk of pancreatitis and pancreatic cancer with liraglutide compared to other antidiabetic drugs. Diabetes Obes Metab. 2014;16:273-5. 
19. Liang C, Bertoia ML, Ding Y, et al. Exenatide use and incidence of pancreatic and thyroid cancer: a retrospective cohort study. Diabetes Obes Metab. 2019;21:1037-42.

20. Morgan CL, Qiao Q, Grandy S, et al. Glucose control and weight change associated with treatment with exenatide compared with basal insulin: a retrospective study. Diabetes Ther. 2018;9:269-83.

21. Pendergrass M, Fenton C, Haffner SM, Chen W. Exenatide and sitagliptin are not associated with increased risk of acute renal failure: a retrospective claims analysis. Diabetes Obes Metab. 2012;14: 596-600.

22. Romley JA, Goldman DP, Solomon M, McFadden D, Peters AL. Exenatide therapy and the risk of pancreatitis and pancreatic cancer in a privately insured population. Diabetes Technol Ther. 2012;14: 904-11.

23. Svanström $H$, Ueda $P$, Melbye $M$, et al. Use of liraglutide and risk of major cardiovascular events: A register-based cohort study in Denmark and Sweden. Lancet Diabetes Endocrinol. 2019;7: 106-14.

24. Tong L, Pan C, Wang H, Bertolini M, Lew E, Meneghini LF. Impact of delaying treatment intensification with a glucagon-like peptide-1 receptor agonist in patients with type 2 diabetes uncontrolled on basal insulin: a longitudinal study of a US administrative claims database. Diabetes Obes Metab. 2018;20:831-9.

25. Wenten M, Gaebler JA, Hussein M, et al. Relative risk of acute pancreatitis in initiators of exenatide twice daily compared with other anti-diabetic medication: a follow-up study. Diabet Med. 2012;29:1412-8.

26. Anyanwagu U, Mamza J, Donnelly R, Idris I. Effect of adding GLP-1RA on mortality, cardiovascular events, and metabolic outcomes among insulintreated patients with type 2 diabetes: a large retrospective UK cohort study. Am Heart J. 2018;196: 18-27.

27. Douros A, Filion $\mathrm{KB}$, Yin $\mathrm{H}$, et al. Glucagon-like peptide-1 receptor agonists and the risk of incident diabetic retinopathy. Diabetes Care. 2018. https:// doi.org/10.2337/dc17-2280.

28. Garg R, Chen W, Pendergrass M. Acute pancreatitis in type 2 diabetes treated with exenatide or sitagliptin. Diabetes Care. 2010;33:2349-54.

29. Hicks BM, Yin H, Yu OHY, Pollak MN, Platt RW, Azoulay L. Glucagon-like peptide-1 analogues and risk of breast cancer in women with type 2 diabetes: population based cohort study using the UK
Clinical Practice Research Datalink. BMJ. 2016. https://doi.org/10.1136/bmj.i5340.

30. Li X, Zhang Z, Duke J. Glucagon-like peptide 1based therapies and risk of pancreatitis: a self-controlled case series analysis. Pharmacoepidemiol Drug Saf. 2014;23:234-9.

31. Nunes AP, Loughlin AM, Qiao Q, et al. Tolerability and effectiveness of exenatide once weekly relative to basal insulin among type 2 diabetes patients of different races in routine care. Diabetes Ther. 2017;8:1349-64.

32. Patorno E, Everett BM, Goldfine AB, et al. Comparative cardiovascular safety of glucagon-like peptide-1 receptor agonists versus other antidiabetic drugs in routine care: a cohort study. Diabetes Obes Metab. 2016;18:755-65.

33. Paul SK, Klein K, Maggs D, Best JH. The association of the treatment with glucagon-like peptide-1 receptor agonist exenatide or insulin with cardiovascular outcomes in patients with type 2 diabetes: a retrospective observational study. Cardiovasc Diabetol. 2015;14:10.

34. Toulis KA, Hanif W, Saravanan P, et al. All-cause mortality in patients with diabetes under glucagonlike peptide- 1 agonists: a population-based, open cohort study. Diabetes Metab. 2017;43:211-6.

35. Velez M, Peterson EL, Wells K, et al. Association of anti-diabetic medications targeting the glucagonlike peptide- 1 pathway and heart failure events in patients with diabetes. J Card Fail. 2015;21:2-8.

36. Ginsberg HN. Insulin resistance and cardiovascular disease. J Clin Invest. 2000;106:453-8.

37. EMA. European Medicines Agency investigates findings on pancreatic risks with GLP-1-based therapies for type-2 diabetes. Press release. 2013. https://www.ema.europa.eu/en/news/europeanmedicines-agency-investigates-findings-pancreaticrisks-glp-1-based-therapies-type-2. Accessed 3 Nov 2020.

38. European Medicines Agency. Ozempic: European public assessment report. 2018. https://www.ema. europa.eu/en/medicines/human/EPAR/ozempic. Accessed 3 Nov 2020.

39. FDA approves first oral GLP-1 treatment for type 2 diabetes. FDA. 2020. https://www.fda.gov/newsevents/press-announcements/fda-approves-firstoral-glp-1-treatment-type-2-diabetes. Accessed 3 Nov 2020.

40. FDA drug safety communication: FDA investigating reports of possible increased risk of pancreatitis and pre-cancerous findings of the pancreas from 
incretin mimetic drugs for type 2 diabetes. FDA. 2019. https://www.fda.gov/drugs/drug-safety-andavailability/fda-drug-safety-communication-fdainvestigating-reports-possible-increased-riskpancreatitis-and-pre. Accessed 3 Nov 2020.

41. GLP-1 receptor agonists: reports of diabetic ketoacidosis when concomitant insulin was rapidly reduced or discontinued. Gov.UK. https://www. gov.uk/drug-safety-update/glp-1-receptor-agonistsreports-of-diabetic-ketoacidosis-whenconcomitant-insulin-was-rapidly-reduced-ordiscontinued. Accessed 3 Nov 2020.

42. Ratner R, Han J, Nicewarner D, Yushmanova I, Hoogwerf BJ, Shen L. Cardiovascular safety of exenatide BID: an integrated analysis from controlled clinical trials in participants with type 2 diabetes. Cardiovasc Diabetol. 2011;10:22.

43. Scheen AJ. Pharmacokinetics and clinical use of incretin-based therapies in patients with chronic kidney disease and type 2 diabetes. Clin Pharmacokinet. 2015;54:1-21.

44. Singh S, Chang H-Y, Richards TM, Weiner JP, Clark JM, Segal JB. Glucagon-like peptide 1-based therapies and risk of hospitalization for acute pancreatitis in type 2 diabetes mellitus: a populationbased matched case-control study. JAMA Intern Med. 2013;173:534-9.
45. Noguchi Y, Katsuno H, Ueno A, et al. Signals of gastroesophageal reflux disease caused by incretinbased drugs: a disproportionality analysis using the Japanese adverse drug event report database. J Pharm Health Care Sci. 2018;4:15.

46. Gudin B, Ladhari C, Robin P, et al. Incretin-based drugs and intestinal obstruction: a pharmacovigilance study. Therapies. 2020;75:641-7.

47. Butler PC, Dry S, Elashoff R. GLP-1-based therapy for diabetes: what you do not know can hurt you. Diabetes Care. 2010;33:453-5.

48. Madsen LW, Knauf JA, Gotfredsen C, et al. GLP-1 receptor agonists and the thyroid: C-cell effects in mice are mediated via the GLP-1 receptor and not associated with RET activation. Endocrinology. 2012;153:1538-47.

49. Henricsson M, Nilsson A, Janzon L, Groop L. The effect of glycaemic control and the introduction of insulin therapy on retinopathy in non-insulin-dependent diabetes mellitus. Diabet Med. 1997;14: 123-31.

50. Pi-Sunyer X, Astrup A, Fujioka $\mathrm{K}$, et al. A randomized, controlled trial of $3.0 \mathrm{mg}$ of liraglutide in weight management. N Engl J Med. 2015;373: 11-22. 